\title{
Prospective Validation of ${ }^{18}$ F-FDG Brain PET Discriminant Analysis Methods in the Diagnosis of Amyotrophic Lateral Sclerosis
}

\author{
Donatienne Van Weehaeghe ${ }^{1}$, Jenny Ceccarini ${ }^{1}$, Aline Delva ${ }^{2}$, Wim Robberecht ${ }^{2,3}$, Philip Van Damme*2-4, \\ and Koen Van Laere*1 \\ ${ }^{I}$ Division of Nuclear Medicine and Molecular Imaging, Department of Imaging and Pathology, University Hospitals Leuven and KU \\ Leuven, Leuven, Belgium; ${ }^{2}$ Department of Neurology, University Hospitals Leuven, Leuven, Belgium; ${ }^{3}$ Department of Neurosciences, \\ Experimental Neurology and Leuven Research Institute for Neuroscience and Disease (LIND), KU Leuven-University of Leuven, \\ Leuven, Belgium; and ${ }^{4}$ Laboratory of Neurobiology, VIB, Vesalius Research Center, Leuven, Belgium
}

\begin{abstract}
An objective biomarker for early identification and accurate differential diagnosis of amyotrophic lateral sclerosis (ALS) is lacking. ${ }^{18} \mathrm{~F}-\mathrm{FDG}$ PET brain imaging with advanced statistical analysis may provide a tool to facilitate this. The objective of this work was to validate volume-ofinterest (VOI) and voxel-based (using a support vector machine [SVM] approach) ${ }^{18} \mathrm{~F}-\mathrm{FDG}$ PET analysis methods to differentiate ALS from controls in an independent prospective large cohort, using a prioriderived classifiers. Furthermore, the prognostic value of ${ }^{18} \mathrm{~F}-\mathrm{FDG}$ PET was evaluated. Methods: A prospective cohort of patients with a suspected diagnosis of a motor neuron disorder $(n=119$; mean age \pm SD, $61 \pm 12$ y; 81 men and 38 women) was recruited. One hundred five patients were diagnosed with ALS (mean age \pm SD, $61.0 \pm 12$ y; 74 men and 31 women) (group 2), 10 patients with primary lateral sclerosis (mean age \pm SD, $55.5 \pm 12$ y; 3 men and 7 women), and 4 patients with progressive muscular atrophy (mean age $\pm S D, 59.2 \pm 5 y$; 4 men). The mean disease duration of all patients was $15.0 \pm 13.4$ mo at diagnosis, with PET conducted $15.2 \pm 13.3$ mo after the first symptoms. Data were compared with a previously gathered dataset of 20 screened healthy subjects (mean age \pm SD, $62.4 \pm 6.4$ y; 12 men and 8 women) and 70 ALS patients (mean age \pm SD, $62.2 \pm 12.5$ y; 44 men and 26 women) (group 1). Data were spatially normalized and analyzed on a VOI basis (statistical software (using the Hammers atlas) and voxel basis using statistical parametric mapping. Discriminant analysis and SVM were used to classify new cases based on the classifiers derived from group 1. Results: Compared with controls, ALS patients showed a nearly identical pattern of hypo- and hypermetabolism in groups 1 and 2. VOI-based discriminant analysis resulted in an $88.8 \%$ accuracy in predicting the new ALS cases. For the SVM approach, this accuracy was $100 \%$. Brain metabolism between ALS and primary lateral sclerosis patients was nearly identical and not separable on an individual basis. Extensive frontotemporal hypometabolism was predictive for a lower survival using a Kaplan-Meier survival analysis $(P<0.001)$. Conclusion: On the basis of a previously acquired training set, ${ }^{18} \mathrm{~F}-\mathrm{FDG}$ PET with advanced discriminant analysis methods is able to accurately distinguish ALS from controls and aids in assessing individual prognosis. Further validation on multicenter datasets and ALS-mimicking disorders is needed to fully assess the general applicability of this approach.
\end{abstract}

Received Sep. 1, 2015; revision accepted Feb. 18, 2016.

For correspondence or reprints contact: Koen Van Laere, Department of Imaging and Pathology, Nuclear Medicine, KU Leuven and UZ Leuven, Herestraat 49, Leuven, Belgium.

E-mail: koen.vanlaere@uzleuven.be

${ }^{*}$ Contributed equally to this work.

Published online Mar. 3, 2016.

COPYRIGHT (c) 2016 by the Society of Nuclear Medicine and Molecular Imaging, Inc.
Key Words: ALS; PET; discriminant analysis; support vector machine; prognosis

J Nucl Med 2016; 57:1238-1243

DOI: 10.2967/jnumed.115.166272

A myotrophic lateral sclerosis (ALS) is an adult-onset motor neuron disorder with predominant involvement of upper and lower motor neurons as well as variable involvement of extramotor areas. The disease causes progressive muscle paralysis, and in about $15 \%$ of cases there is clinically apparent cognitive impairment. ALS, with both upper and lower motor neuron involvement, is part of the spectrum of motor neuron diseases consisting also of primary lateral sclerosis (PLS), with exclusively upper motor neuron disease, and progressive muscular atrophy (PMA), with only lower motor neuron disease. Both PMA and PLS can progress to ALS (1-4).

The diagnosis of ALS predominantly relies on clinical parameters. Upper motor neuron loss causes spasticity and hyperreflexia, whereas lower motor neuron loss leads to muscle weakness and muscle wasting with fasciculations. ALS may be separated into bulbar- or spinal-onset disease, depending on the site of the onset of the first symptom. The clinical diagnostic criteria have been revised to improve the sensitivity in 2 steps, with both the revised El Escorial and the Awaji-Shima diagnostic criteria being used in clinical practice $(5,6)$.

Recently, ${ }^{18}$ F-FDG PET showed promising results in differentiating subjects with motor neuron disorders from healthy controls. In patients with ALS, a pattern of relative hypometabolism in the primary motor cortex, premotor cortices, and supplementary motor cortex but extending to extramotor areas such as the frontal and parietal lobes as well has been observed (2,4,7-11). Relative hypermetabolism is observed in the mesotemporal cortex, cerebellum, and upper brain stem $(2,4,7,10,12-14)$. Within-center retrospective discriminant analysis methods to differentiate subjects with early ALS from controls have resulted in an overall classification accuracy of $90 \%-95 \%(2,4,10)$.

Other MR-based imaging techniques, especially diffusion tensor imaging, MR spectroscopy, and voxel-based morphometry, have been used to separate ALS patients from healthy controls. The best results in single-center studies have been obtained by combined MR 
imaging approaches $(15,16)$. Combined diffusion tensor imaging and spectroscopy resulted in a sensitivity of $93 \%$ and a specificity of $85 \%$ (17), whereas diffusion tensor imaging in combination with voxel-based morphometry resulted in a sensitivity of $92 \%$ and a specificity of $88 \%$ (18). However, no replication of these studies has been performed so far.

To use an imaging biomarker approach in a general clinical setting, an independent validation of previously gathered discriminant markers is needed. To prospectively assess the diagnostic accuracy of ${ }^{18}$ F-FDG PET imaging in a new set of early diagnosed patients with a suspected diagnosis of ALS within-center, the main aim of this study was to use a priori classifiers from both a volumeof-interest (VOI) and a voxelwise-based support vector machine (SVM) discriminant analysis in a novel large prospectively acquired group of ALS patients.

Furthermore, because previous studies have indicated that ${ }^{18}$ F-FDG PET may be useful to obtain prognostic information on survival, in which frontotemporal hypometabolism is directly associated with a lower survival rate $(4,19,20)$, our second aim was to objectively assess semiquantitative parameters of frontotemporal hypometabolism to predict survival.

\section{MATERIALS AND METHODS}

\section{Patients}

A new, prospective set of patients with a suspected diagnosis of $\operatorname{ALS}(n=119 ;$ mean age $\pm \mathrm{SD}, 61.0 \pm 12 \mathrm{y} ; 81$ men and 38 women) was recruited from the tertiary neuromuscular clinic at the University Hospital Leuven (Belgium) between October 2012 and January 2015 (Table 1). In 105 patients, the diagnosis of ALS was confirmed (group 2 , mean age $\pm \mathrm{SD}, 61.7 \pm 12 \mathrm{y} ; 74$ men and 31 women), 10 patients were diagnosed with PLS (mean age \pm SD, $55.5 \pm 12 \mathrm{y} ; 3$ men and 7 women), and 4 patients were diagnosed with PMA (mean age \pm SD, $59.2 \pm 5 \mathrm{y} ; 4$ men). For this validation study, the small group of PMA patients was excluded. Table 1 summarizes the demographic and disease characteristics of the ALS and PLS patients.

Disease onset was determined by the patient's recollection of the month in which the initial symptoms of muscle weakness or dysarthria/ dysphagia occurred. The mean disease duration for ALS group 2 was $15.0 \pm 13.4$ mo at diagnosis. ${ }^{18} \mathrm{~F}-\mathrm{FDG}$ PET was planned at the initial visit and performed on average $15.2 \pm 13.3$ mo after first symptom onset. All patients had a negative history of other neurologic disorders. All patients were investigated neurologically and were electrodiagnostically tested as part of their clinical workup by an experienced specialist in neuromuscular disorders. Both the revised El Escorial and the Awaji-Shima criteria were applied $(5,6)$. There was no evidence of respiratory distress or nutritional abnormalities, such as dehydration or ketosis, at the time of the ${ }^{18} \mathrm{~F}-\mathrm{FDG}$ PET scan. The revised version of the ALS functional rating scale was used. Genetic testing was performed on most patients but not considered for this study. Patients of the training set (group $1, n=70$; mean age $\pm \mathrm{SD}, 62.1 \pm 12.5 \mathrm{y} ; 44$ men and 26 women; Table 1) were recruited at the same center between January 2011 and January 2013, using the same inclusion/exclusion criteria. As for the previous study, genetic testing results needed to be included (4), thus, there were a few months of recruitment time overlap between both training and new group. The 20 control subjects (mean age $\pm \mathrm{SD}$, $62.4 \pm 6.4 \mathrm{y} ; 12$ men and 8 women; Table 1) were part of a screened reference database set of 30 carefully screened healthy volunteers aged between 50 and $80 \mathrm{y}$, as described before (4), and randomly selected to obtain age and sex matching to ALS groups 1 and 2. The ALS group 1 $(n=70)$ and the control subjects $(n=20)$ were the same as used in a previous study (4). The current study was approved by the local University Hospital Ethics Committee, and all subjects gave written informed consent.

\section{PET Acquisition and Reconstruction}

To standardize acquisition, all subjects fasted at least $6 \mathrm{~h}$ before the ${ }^{18} \mathrm{~F}-\mathrm{FDG}$ PET acquisition. Before ${ }^{18} \mathrm{~F}-\mathrm{FDG}$ injection, blood glucose was measured. For group 2, the mean glycemia was $104 \pm 15 \mathrm{mg} / \mathrm{dL}$

TABLE 1

Patient Characteristics Among Controls, Training Set (ALS Group 1), and New Dataset (ALS Group 2)

\begin{tabular}{|c|c|c|c|c|}
\hline Characteristic & Control & $\begin{array}{c}\text { ALS group } 1 \\
\text { (training set [no PLS/PMA]) }\end{array}$ & $\begin{array}{c}\text { ALS group } 2 \\
\text { (new dataset [no PLS/PMA]) }\end{array}$ & PLS \\
\hline$n$ (total) & 20 & 70 & 105 & 10 \\
\hline Age $(y)$ & $62.4 \pm 6.4$ & $62.1 \pm 12.5$ & $61.7 \pm 12.0$ & $55.5 \pm 12.0$ \\
\hline \multicolumn{5}{|l|}{ Sex } \\
\hline Male & 12 & 44 & 74 & 3 \\
\hline Female & 8 & 26 & 31 & 7 \\
\hline Onset (mo) & - & $15.2 \pm 10.7$ & $15.2 \pm 13.3$ & - \\
\hline Awaji-Shima & - & & & - \\
\hline Definite & & 32 & 28 & \\
\hline Probable & & 29 & 52 & \\
\hline Possible & & 9 & 25 & \\
\hline Onset type & - & & & - \\
\hline Bulbar & & 21 & 23 & \\
\hline Spinal & & 48 & 82 & \\
\hline $\begin{array}{l}\text { Forced expiratory vital } \\
\text { capacity (\%) }\end{array}$ & - & $94.7 \pm 23.3$ & $84.1 \pm 26.6$ & - \\
\hline ALS functional rating scale & - & $36.3 \pm 7.2(n=46)$ & $33.7 \pm 9.0(n=105)$ & - \\
\hline
\end{tabular}

Values are mean \pm SD 
(range, $72-189 \mathrm{mg} / \mathrm{dl}$ ). ${ }^{18} \mathrm{~F}-\mathrm{FDG}$ PET scans were acquired using an ECAT HR + camera $(n=99)$ (Siemens) or HiRez PET-CT camera $(n=6)$ (Siemens) operated in 3-dimensional mode. ${ }^{18} \mathrm{~F}-\mathrm{FDG}(151 \pm$ $8 \mathrm{MBq}$ ) was injected intravenously under standard conditions, that is, subjects lying supine in a dimly lit, quiet room, with ears and eyes open. Thirty minutes after ${ }^{18} \mathrm{~F}-\mathrm{FDG}$ injection, a dynamic scan of $30 \mathrm{~min}$ ( 6 frames of 5 min each) was started. During the acquisition, the subject's head was immobilized by means of a vacuum pillow. On the HR+ camera, attenuation- and scatter-corrected ${ }^{18} \mathrm{~F}$-FDG images were reconstructed using 3-dimensional filtered back projection with a Hanning postfilter, resulting in a full width at half maximum of $6 \mathrm{~mm}$. On the HiRez, ${ }^{18} \mathrm{~F}-\mathrm{FDG}$ images were reconstructed using iterative ordered-subset expectation maximization (4 iterations, 4 subsets).

\section{F-FDG PET Image Analysis}

All reconstructed data were analyzed quantitatively in 2 ways. First, a VOI analysis was performed with an automatic VOI-analysis protocol using the PNEURO tool in PMOD (version 3.4; PMOD Inc.) with which a total of 83 individual brain VOIs were obtained according to the Hammers N30R83 maximum probability atlas. Relative metabolic activity was calculated by dividing each individual VOI activity by the individual average gray matter activity.

Second, a voxel-based analysis was conducted using statistical parametric mapping (SPM8; Wellcome Trust Centre for Neuroimaging), implemented in Matlab (R2012b; The MathWorks Inc.). All scans were spatially normalized to Montreal Neurological Institute space using the SPM PET template and nonrigid registration with 16 iterations and isotropic Gaussian smoothing with a full width at half maximum of $8 \mathrm{~mm}$. The dimensions of the resulting voxels were $2 \times 2 \times 2 \mathrm{~mm}$. Data were normalized to the average gray matter activity of each image using proportional scaling.

Because there were no significant age and gender differences between groups, the analyses were conducted without age and gender as nuisance variables. Because some patients $(n=6)$ were scanned on another PET camera, camera type was included as a nuisance variable. Unless specified otherwise, for SPM group comparisons a $t$ test was performed with a threshold $p_{\text {height }}$ (the chance of finding a peak with this or a greater glucose hypo- or hyper-metabolism under the null hypothesis) (uncorrected) of less than 0.001 and minimum extent threshold set at a $\mathrm{k}_{\mathrm{E}}$ (cluster extent threshold, only clusters with at least $\mathrm{k}_{\mathrm{E}}$ voxels are retained [here 20 voxels]) of 20 ( $P$ cluster $<0.05$; false-discovery ratecorrected). The $\mathrm{k}_{\mathrm{E}}$ threshold was used to be consistent with the previous study (4) and to allow detection of potential small clusters in motor areas of the corticospinal and central gray nuclei.

\section{Prognosis: Visual and Semiquantitative Analysis}

All ${ }^{18}$ F-FDG PET scans were first evaluated visually with routinely used clinical analysis and visualization software (MIMVista; MIMSoftware Inc.), with spatially normalized transverse slices, surface projections, and $\mathrm{z}$ map renderings by comparison to normal data, with a threshold at -2 SDs. The $\mathrm{z}$ images were then visually scored for frontotemporal involvement by 1 observer. Consistent with previous work (4), patients with uptake below 2 SDs in one fifth or more of a frontal lobe or one half or more of an anterior temporal lobe were classified as having extensive frontotemporal involvement. The total ALS group (= training dataset ALS group 1 plus the new dataset ALS group 2) was thereby dichotomized into a group with extensive frontotemporal involvement $(n=34)$ and patients without extensive involvement (i.e., mild or absent involvement) $(n=141)$. An updated survival analysis (Kaplan-Meier) was performed for the total set of ALS patients using this dichotomization. Survival was calculated as the time when the first symptoms of weakness appeared until the time of death.

To further assess the correlation between survival and frontotemporal metabolism more parametrically, the volume-weighted average VOI-based relative ${ }^{18} \mathrm{~F}$-FDG activity in the frontotemporal areas (Supplemental Fig. 1; supplemental materials are available at http://jnm.snmjournals. org) of the 80 deceased ALS patients (from the training and new dataset) was calculated. A linear correlation of the volume-weighted average versus the survival was performed. As an index, a volumeweighted average of frontal and anterior temporal relative activity was calculated, normalized to whole-brain activity.

\section{Statistics}

Standard statistics were conducted using Statistica (version 12.0; Statsoft). Significance was considered at a $P$ value of less than 0.05 after Bonferroni correction for the VOI-based analysis. Conventional discriminant analysis between the training set (ALS group 1, $n=70$ ) and controls was done using the above-defined 83 VOI regions and was specifically computed in SPSS (version 22.0; IBM), with the leave-one-out technique for cross-validation. A forward stepwise analysis was done, using Wilks' $\lambda$ method and standard $F$ values for an entry of 3.84 and removal of 2.71. As a conservative estimate, prior probabilities were considered as equal instead of computed by group size. The classifiers of this approach were then used to classify the new dataset (ALS group 2, $n=105$ ), and the new dataset with inclusion of PLS patients, using the scoring wizard in SPSS. Finally, also a 3-way discriminant analysis was done using all ALS patients, PLS, and controls, to investigate the possibility to separate ALS and PLS patients.

Second, voxel-based SVM approach was also conducted (21). Also here, the classifiers of the SVM approach obtained in our previous study on ALS group 1 (4) were used to investigate the ability to classify ALS group 2. An SVM with a linear kernel using the default soft margin option (Spider version 1.71 with Matlab version 7.9; http://people.kyb.tuebingen.mpg.de/spider/) was used to classify each subject. The brain mask was defined by those voxels exceeding $50 \%$ of the mean of the ${ }^{18} \mathrm{~F}-\mathrm{FDG}$ PET images, and the same mask was used in all analyses.

\section{RESULTS}

\section{Group Comparison (Training Set and New ALS Patients Versus Controls)}

A voxel-based comparison of the ALS patients of both ALS group 1 and 2 resulted in visually nearly identical results for the SPM analysis, shown in Figure 1. Significant symmetric hypometabolism

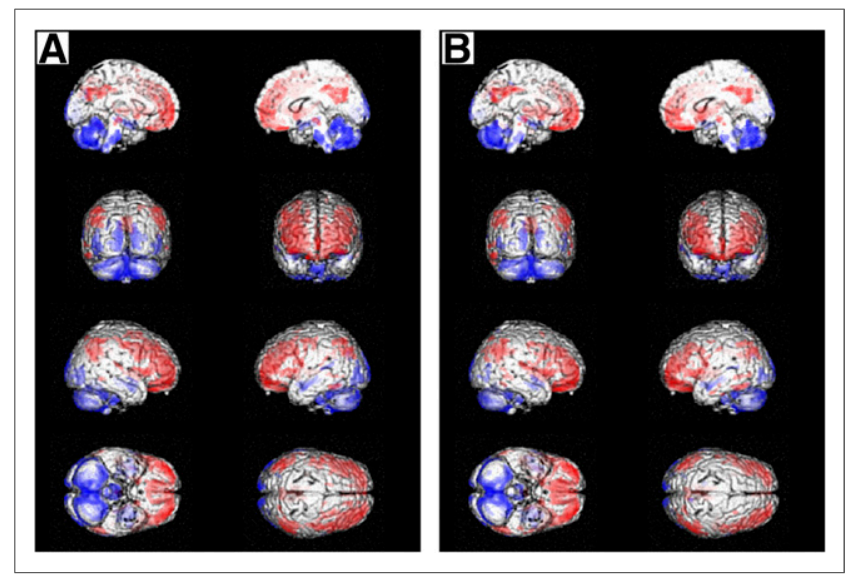

FIGURE 1. Statistical parametric mapping results with surface and interhemispheric cluster projections showing relative hypo- and hypermetabolism (red and blue, respectively), for original training set (ALS group $1, n=70$ ) versus controls (4) (A) and prospectively acquired dataset of ALS patients (ALS group 2, $n=105$ ) (B). Clusters are shown at $\mathrm{p}_{\text {height }}<0.001$ and extent threshold $\mathrm{k}_{\mathrm{E}}=20$ voxels. 
was seen in the frontal cortex, premotor cortex, and inferolateral part of the parietal cortex, whereas clusters of symmetric relative hypermetabolism were seen in the occipital cortical pole (primary visual cortex), cerebellum, upper brain stem, and medial temporal cortex. For PLS patients, a similar distribution was found but only at low significance $p_{\text {height }}$ threshold (data not shown).

\section{VOI-Based Discriminant Analysis}

Using the a priori classifiers of ALS group 1 versus controls, the classification of the new dataset (ALS group 2) resulted in a sensitivity of $88.6 \%$, a specificity of $90.0 \%$, and an accuracy of $88.8 \%$. Twelve ALS patients from ALS group 2 were misclassified into the control group, and 2 controls were misclassified into the ALS group (Table 2).

The most discriminating gray matter regions for the classifiers were the midfrontal, precentral, and superior frontal gyrus and the posterior cingulum.

When the PLS patients were analyzed using the a priori classifiers, 9 of 10 PLS patients were classified into the ALS group, in accordance with the similar metabolic profile. When a 3-way discriminant analysis including PLS as a third group and all ALS patients was used, sensitivity for ALS detection decreased to $77.7 \%$ because of 24 misclassifications into the PLS group, whereas only 6 PLS patients were classified into the ALS group, and only 1 control subject was misclassified (Supplemental Table 1), again illustrating the high similarity between the PLS and ALS metabolic profile.

\section{SVM Analysis}

On the basis of the ALS group 1 training set, the ALS group 2 cases were perfectly classified (sensitivity/specificity/accuracy all $100 \%)$. Performing SVM on the whole ALS group $(n=175)$ resulted in a sensitivity of $100 \%$, a specificity of $95 \%$, and an accuracy of $95 \%$, where 1 healthy control was falsely classified as an ALS patient. The feature weights of the classifier of the new ALS group 2 (Fig. 2) showed high similarity with the previously published group 1 classifier (4). All PLS cases were also classified into the ALS pattern using this training set for a 2-way SVM ALS versus control classification with PLS patients.

\section{Survival Analysis}

To estimate the prognostic value of the extent of hypometabolism in ALS patients, 2 analyses were performed. A qualitative visual scoring of the $\mathrm{z}$ images was used to categorize patients into patients with or without extensive frontotemporal hypometabolism. A Kaplan-Meier survival showed worse prognosis in the group with extensive hypometabolism in the frontal or anterior

TABLE 2

VOI-Based Classification Matrix of ALS Group 2 Versus Controls Using A Priori Classifiers of ALS Group 1 Versus Controls

\begin{tabular}{lccc}
\hline Group & \% correct & Controls & ALS \\
\hline Controls & 90.0 & 18 & 2 \\
ALS & 88.6 & 12 & 93 \\
Total & 88.8 & 30 & 95 \\
& & & \\
\hline CON $=$ controls. & & & \\
\hline
\end{tabular}

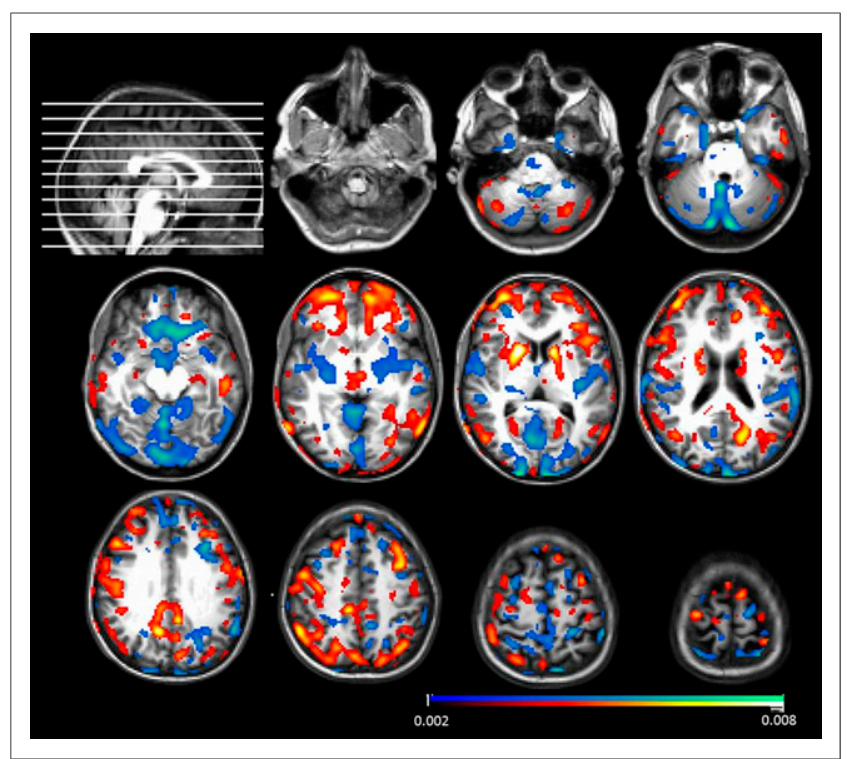

FIGURE 2. Feature weights of classifier for ALS group $2(n=105)$ vs. healthy control cases $(n=20)$ projected onto normalized structural MR image in Montreal Neurologic Institute space. Clusters indicate areas with high discriminative impact based on relative hypometabolism (yellowred) and relative hypermetabolism (blue). Scale of feature weights represents how much a voxel contributes. Scale was normalized so that sum of all weights is 1 . Only voxels with weight of more than 0.002 in absolute value are shown.

temporal regions $(n=34)$ compared with the remainder of the ALS patients $(n=141)$ (mean survival $=20.1 \mathrm{mo}$ vs. $51.3 \mathrm{mo}$, $P<0.001$ ) (Fig. 3).

To obtain quantitative correlations between survival times and measures of frontotemporal hypometabolism, the volume-weighted averaged frontotemporal hypometabolism index was calculated for each patient. The linear correlation between survival and volumeweighted average did not, however, reach significance $(P=0.13)$, possibly because of the relatively small numbers of patients with significant hypometabolism in the frontotemporal lobes in the studied population.

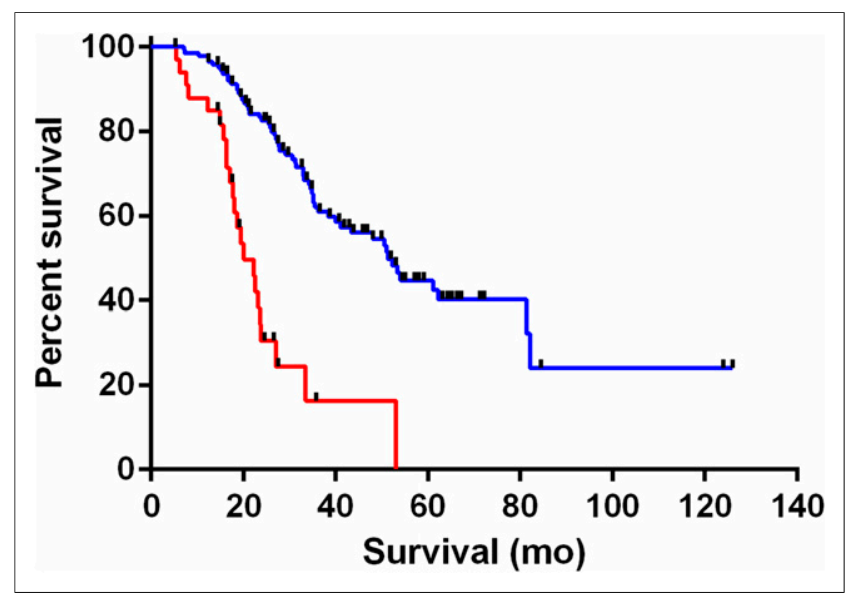

FIGURE 3. Kaplan-Meier survival plot of all ALS patients (ALS group $1+$ ALS group 2) with (red line, $n=34$ ) and without (blue line, $n=$ 141) extensive hypometabolism in frontal or temporal cortex $(n=175$, $P<0.001)$. 


\section{DISCUSSION}

In this monocentric validation study, we found that VOI- and voxel-based discriminant analysis of ALS versus controls with a priori-defined classifiers using an extended training set showed good classification results for new cases, with an accuracy of $89 \%$ and $100 \%$, respectively. The regions found to be most discriminating (hypometabolism in the prefrontal cortex and premotor cortex; relative hypermetabolism in the cerebellum, upper brain stem, and the medial temporal cortex) are in line with previous findings $(4,8,10,22)$ and with the known pathophysiology of ALS. ALS is characterized by damage to large pyramidal cells in the primary motor cortex, brain stem motor nuclei, and spinal $\alpha$-motorneurons (stage 1). On progression, the disease can spread to additional motor neuron areas (stage 2), the prefrontal cortex (stage 2-3), and the anterior temporal cortex (stage 4) through the corticospinal, corticopontine, corticorubral, and corticostriatal tracts, with finally hippocampal involvement $(23,24)$. Whereas hypometabolism in the frontal cortex may be a direct consequence of this involvement, the relative hypermetabolism in the brain stem and medial temporal cortex has been attributed to possible neuroinflammation with activated astrocytes and microglia and therefore upregulated glucose metabolism (12). In contrast to the findings of Pagani et al. (10), we found a systematic slight relative hypermetabolism in the primary visual cortex, which may be confounded by a difference in the way the control data were gathered (eyes closed).

The high numbers in discriminant accuracy of both analysis methods indicate a consistency in recruitment (only patients with clinical suspicion for ALS were scanned) and clinical diagnostic parameters. The nearly identical results in ALS groups 1 and 2 compared with controls indicates that the use of El Escorial and Awaji-Shima diagnostic criteria leads to reproducible identification of patients with a particular cerebral metabolic signature. The high accuracy of the current approach may allow extension to an early-stage detection of ALS, but this was not investigated in the current study. However, this within-center validation is only a first step to evaluate the robustness of ${ }^{18} \mathrm{~F}-\mathrm{FDG}$ as a biomarker as adjunct in the diagnosis of ALS. Because acquisition and image processing factors (e.g., injected activity, acquisition procedure, attenuation correction, resolution, and scatter correction) may introduce additional variability and bias, it is important to further validate this approach with data from other centers. To become a reliable test, an accuracy of at least $80 \%$ should be reached (5). The implications of such differences in camera, acquisition, and processing parameters on classification accuracy need to be investigated in more detail, and at this moment crossvalidation of our data with the large Italian database is under way.

As for specificity, ALS and PLS patients show a similar metabolic profile, and direct VOI-based discriminant analysis is not able to separate both groups with reasonable accuracy, confirming that a similar ${ }^{18}$ F-FDG PET brain pattern is present in both types of patients that does not allow physicians to diagnose ALS and PLS patients on an individual level using ${ }^{18} \mathrm{~F}$-FDG PET, which is consistent with previous findings (4). To further study the specificity of the findings, a direct comparison with ALS-mimicking disorders and frontotemporal dementia patients should be established. However, as only patients with a clinical suspicion of ALS undergo ${ }^{18} \mathrm{~F}-\mathrm{FDG}$ PET, the results obtained reflect the situation in daily clinical practice, and the technique can be useful on an individual patient level to discriminate between ALS/PLS and controls. Moreover, PET can presumably help in the early detection of ALS, because metabolic changes occur before structural and clinical abnormalities. The technique can be applied in a clinical setting when the a priori datasets and SVM routines are available and takes only an additional few minutes then to be calculated.

The SVM approach has been used in other neurodegenerative disorders for individual patient classification. For example, in the setting of dementia, the accuracy of SVM and ${ }^{18}$ F-FDG PET to differentiate between patients with mild cognitive impairment that will convert to AD and controls was $83.0 \%$ using only ${ }^{18} \mathrm{~F}-\mathrm{FDG}$ PET (25). On the basis of the Alzheimer Disease Neuroimaging Initiative database, the combination of ${ }^{18} \mathrm{~F}-\mathrm{FDG}$ PET and volumetric MR resulted in an accuracy of $91 \%$ to classify AD patients from controls (26). In the differential diagnosis of dementing disorders, Tahmasian et al. studied the ability of combined ${ }^{18} \mathrm{~F}-\mathrm{FDG}$ PET and MR with SVM and found accuracies of $77.5 \%$ for AD, $82.5 \%$ for frontotemporal dementia, $97.5 \%$ for semantic dementia, and $87.5 \%$ for progressive nonfluent aphasia (27). The magnitude of accuracies in this setting using either ${ }^{18} \mathrm{~F}-\mathrm{FDG}$ or ${ }^{18} \mathrm{~F}-\mathrm{FDG}$ plus MR information is in line with our current results for ALS and provide an avenue to optimize the diagnostic performance of ${ }^{18}$ F-FDG PET. To our knowledge, no studies combining ${ }^{18} \mathrm{~F}$-FDG PET and MRI have been published to further refine early diagnosis of ALS, which may become better feasible with the increase in simultaneous PET/MR cameras worldwide.

In our study, we confirmed that frontotemporal hypometabolism is associated with a worse individual prognosis, likely because of an underlying frontotemporal dementia or cognitive/behavioral dysfunction, a finding already reported in previous studies (1922,28). In this study, there is a significant difference in survival between patients with and without extensive frontotemporal hypometabolism on a visual basis but we were not able to find a significant linear correlation between the survival and the volumeweighted average of frontotemporal ${ }^{18} \mathrm{~F}-\mathrm{FDG}$ activity. This may be because of the finding that most ALS patients have only small decreases in frontotemporal metabolism, which may have no impact on survival.

There are several limitations to this study. First, no 3-dimensional MRI was available, so no correction for partial-volume effects to correct for atrophy was applied (29-32). However, because glucose uptake is a combined measure of implicit cellular function and macroscopic atrophy effects, the validity and diagnostic power remains unchanged. Second, both right and left hemisphere VOI values were taken together because a symmetric involvement of brain regions was demonstrated previously (33). This could cause some loss of discriminatory information in asymmetrically involved regions (34). In the SPM results, however, little asymmetry was found in the group differences, therefore justifying a data reduction approach by combining contralateral VOIs. Third, this prospective study does not include longitudinal imaging, therefore, the importance of timing and disease stage on discrimination accuracy could not be established. Most of our patients had a confirmed diagnosis of ALS when undergoing ${ }^{18}$ F-FDG PET so they were not in the early phases of the disease. Future studies are necessary to determine the discrimination accuracy in the very early stage of ALS diagnosis near first symptoms.

Regarding the prognostic aspect with the visual analysis, the threshold for visual dichotomization of extensive versus mild frontotemporal involvement was chosen arbitrarily, taking clinical expertise into account to avoid too high false-positives or falsenegatives. In this study, no sensitivity analysis of this threshold 
was conducted, which could result in a better discrimination of poor survivors.

Finally, the same group of healthy controls was used for both the training and the validation set. Use of the same group may theoretically give rise to systematic bias, so further studies should take the robustness of the control group into account using a new or extended control group or using cross-validation with multicenter control data.

\section{CONCLUSION}

The current study validates in an independent prospectively acquired group of ALS patients that VOI- or voxel-based discriminant analysis using a priori-defined classifiers with ${ }^{18} \mathrm{~F}$-FDG PET is a promising tool on an individual patient level to differentiate ALS from controls and thereby aid in early diagnosis. Furthermore, these data show that extensive frontotemporal hypometabolism is related to poorer survival.

\section{DISCLOSURE}

The costs of publication of this article were defrayed in part by the payment of page charges. Therefore, and solely to indicate this fact, this article is hereby marked "advertisement" in accordance with 18 USC section 1734 . This work was supported by a grant from the KU Leuven (GOA/11/014), by the Interuniversity Attraction Poles (IUAP) program P7/16 of the Belgian Federal Science Policy Office, by the FWO-Vlaanderen under the frame of E-RARE-2, by the ERA-Net for Research on Rare Diseases (PYRAMID), and by a EU Joint Programme-Neurodegenerative Disease Research (JPND) project (STRENGTH). Koen Van Laere and Philip Van Damme are senior clinical investigators of the Flemish Fund for Scientific Research (FWO-Vlaanderen). Philip Van Damme is supported by the Belgian ALS League. Jenny Ceccarini is a postdoctoral fellow of the FWO. No other potential conflict of interest relevant to this article was reported.

\section{ACKNOWLEDGMENTS}

We acknowledge the skilled help of the radiopharmacy and medical physics team at UZ Leuven (Marva Bex; Tjibbe de Groot, PhD; Kim Serdons, Pharm PhD; Kristof Baete, PhD; Michel Koole, $\mathrm{PhD}$; Johan Nuyts, Ir PhD; and Andrey Postnov, PhD).

\section{REFERENCES}

1. Rowland LP, Shneider NA. Amyotrophic lateral sclerosis. $N$ Engl J Med. 2001;344:1688-1700.

2. Quartuccio N, Van Weehaeghe D, Cistaro A, Jonsson C, Van Laere K, Pagani M. Positron emission tomography neuroimaging in amyotrophic lateral sclerosis: what is new? Q J Nucl Med Mol Imaging. 2014;58:344-354.

3. Renton AE, Chio A, Traynor BJ. State of play in amyotrophic lateral sclerosis genetics. Nat Neurosci. 2014;17:17-23.

4. Van Laere K, Vanhee A, Verschueren J, et al. Value of 18fluorodeoxyglucosepositron-emission tomography in amyotrophic lateral sclerosis: a prospective study. JAMA Neurol. 2014;71:553-561.

5. Costa J, Swash M, de Carvalho M. Awaji criteria for the diagnosis of amyotrophic lateral sclerosis:a systematic review. Arch Neurol. 2012;69:1410-1416.

6. Schrooten M, Smetcoren C, Robberecht W, Van Damme P. Benefit of the Awaji diagnostic algorithm for amyotrophic lateral sclerosis: a prospective study. Ann Neurol. 2011;70:79-83.

7. Cistaro A, Cuccurullo V, Quartuccio N, Pagani M, Valentini MC, Mansi L. Role of PET and SPECT in the study of amyotrophic lateral sclerosis. Biomed Res Int. 2014;2014:237437.

8. Dalakas MC, Hatazawa J, Brooks RA, Di Chiro G. Lowered cerebral glucose utilization in amyotrophic lateral sclerosis. Ann Neurol. 1987;22:580-586.
9. Ludolph AC, Langen KJ, Regard M, et al. Frontal lobe function in amyotrophic lateral sclerosis: a neuropsychologic and positron emission tomography study. Acta Neurol Scand. 1992;85:81-89.

10. Pagani M, Chio A, Valentini MC, et al. Functional pattern of brain FDG-PET in amyotrophic lateral sclerosis. Neurology. 2014;83:1067-1074.

11. Pringle CE, Hudson AJ, Munoz DG, Kiernan JA, Brown WF, Ebers GC. Primary lateral sclerosis: clinical features, neuropathology and diagnostic criteria. Brain. 1992;115:495-520.

12. Cistaro A, Valentini MC, Chio A, et al. Brain hypermetabolism in amyotrophic lateral sclerosis: a FDG PET study in ALS of spinal and bulbar onset. Eur J Nucl Med Mol Imaging. 2012;39:251-259.

13. Corcia P, Tauber C, Vercoullie J, et al. Molecular imaging of microglial activation in amyotrophic lateral sclerosis. PLoS One. 2012;7:e52941.

14. Turner MR, Cagnin A, Turkheimer FE, et al. Evidence of widespread cerebral microglial activation in amyotrophic lateral sclerosis: an $\left[{ }^{11} \mathrm{C}\right](\mathrm{R})-\mathrm{PK} 11195$ positron emission tomography study. Neurobiol Dis. 2004;15:601-609.

15. Foerster BR, Dwamena BA, Petrou M, et al. Diagnostic accuracy of diffusion tensor imaging in amyotrophic lateral sclerosis: a systematic review and individual patient data meta-analysis. Acad Radiol. 2013;20:1099-1106.

16. Pradat PF, El Mendili MM. Neuroimaging to investigate multisystem involvement and provide biomarkers in amyotrophic lateral sclerosis. Biomed Res Int. 2014;2014:467560.

17. Foerster BR, Carlos RC, Dwamena BA, et al. Multimodal MRI as a diagnostic biomarker for amyotrophic lateral sclerosis. Ann Clin Transl Neurol. 2014;1: 107-114.

18. Filippini N, Douaud G, Mackay CE, Knight S, Talbot K, Turner MR. Corpus callosum involvement is a consistent feature of amyotrophic lateral sclerosis. Neurology. 2010;75:1645-1652.

19. Chiò A, Ilardi A, Cammarosano S, Moglia C, Montuschi A, Calvo A. Neurobehavioral dysfunction in ALS has a negative effect on outcome and use of PEG and NIV. Neurology. 2012;78:1085-1089.

20. Elamin M, Bede P, Byrne S, et al. Cognitive changes predict functional decline in ALS: a population-based longitudinal study. Neurology. 2013;80:1590-1597.

21. Vandenberghe R, Nelissen N, Salmon E, et al. Binary classification of ${ }^{18} \mathrm{~F}-$ flutemetamol PET using machine learning: comparison with visual reads and structural MRI. Neuroimage. 2013;64:517-525.

22. Abrahams S, Goldstein LH, Kew JJ, et al. Frontal lobe dysfunction in amyotrophic lateral sclerosis: a PET study. Brain. 1996;119:2105-2120.

23. Fatima M, Tan R, Halliday GM, Kril JJ. Spread of pathology in amyotrophic lateral sclerosis: assessment of phosphorylated TDP-43 along axonal pathways. Acta Neuropathol Commun. 2015;3:47-55.

24. Kassubek J, Muller HP, Del Tredici K, et al. Diffusion tensor imaging analysis of sequential spreading of disease in amyotrophic lateral sclerosis confirms patterns of TDP-43 pathology. Brain. 2014;137:1733-1740.

25. Pagani M, De Carli F, Morbelli S, et al. Volume of interest-based $\left[{ }^{18} \mathrm{~F}\right]$ fluorodeoxyglucose PET discriminates MCI converting to Alzheimer's disease from healthy controls: a European Alzheimer's Disease Consortium (EADC) study. Neuroimage Clin. 2014;7:34-42.

26. Dukart J, Mueller K, Barthel H, et al. Meta-analysis based SVM classification enables accurate detection of Alzheimer's disease across different clinical centers using FDG-PET and MRI. Psychiatry Res. 2013;212:230-236.

27. Tahmasian M, Shao J, Meng C, et al. Based on the network degeneration hypothesis: separating individual patients with different neurodegenerative syndromes in a preliminary hybrid PET/MR study. J Nucl Med. 2016;57: 410-415.

28. Irwin DJ, McMillan CT, Brettschneider J, et al. Cognitive decline and reduced survival in C9orf72 expansion frontotemporal degeneration and amyotrophic lateral sclerosis. J Neurol Neurosurg Psychiatry. 2013;84:163-169.

29. Agosta F, Gorno-Tempini ML, Pagani E, et al. Longitudinal assessment of grey matter contraction in amyotrophic lateral sclerosis: a tensor based morphometry study. Amyotroph Lateral Scler. 2009;10:168-174.

30. Chang JL, Lomen-Hoerth C, Murphy J, et al. A voxel-based morphometry study of patterns of brain atrophy in ALS and ALS/FTLD. Neurology. 2005;65:75-80.

31. Grosskreutz J, Kaufmann J, Fradrich J, Dengler R, Heinze HJ, Peschel T. Widespread sensorimotor and frontal cortical atrophy in amyotrophic lateral sclerosis. BMC Neurol. 2006;6:17-26.

32. Tsujimoto M, Senda J, Ishihara T, et al. Behavioral changes in early ALS correlate with voxel-based morphometry and diffusion tensor imaging. J Neurol Sci. 2011;307:34-40.

33. Bede P, Hardiman O. Lessons of ALS imaging: pitfalls and future directions-a critical review. Neuroimage Clin. 2014;4:436-443.

34. Turner MR, Wicks P, Brownstein CA, et al. Concordance between site of onset and limb dominance in amyotrophic lateral sclerosis. J Neurol Neurosurg Psychiatry. 2011;82:853-854. 